\title{
Mit dem Gewicht steigt das Arteriosklerose-Risiko
}

Fragestellung: Ist eine Gewichtszunahme im Laufe einer intensiven Insulintherapie mit dem Auftreten einer subklinischen Arteriosklerose assoziiert?

Hintergrund: Bei einer intensiven Insulintherapie nehmen Diabetiker oft stark an Gewicht zu. Unklar ist, wie bedeutsam die Gewichtszunahme ist. Nun liegt erstmals eine kontrollierte Studie vor, in der geprüft wird, ob die Gewichtszunahme mit der Entwicklung einer subklinischen Arteriosklerose assoziiert ist.

Patienten und Methodik: In der Epidemiology of Diabetes Interventions and Complications(EDIC)-Studie, in der nach Beendigung der DCCT-Studie 1168 Typ-1-Diabetiker (über 18 Jahre) betreut werden, untersuchte man das Arterioskleroserisiko und die klinischen Endpunkte. Die Patienten wurden in der 1983 begonnenen DCCT-Studie randomisiert zu einer konventionellen (1-2 Injekt./d) oder einer intensiven Insulintherapie (ICT, $\geq 3$ Injekt./d). In EDIC ermittelte man nach einem Jahr und sechs Jahren die Intima-Media-Dicke (IMT) und nach acht Jahren den koronararteriellen Kalziumscore. Die Zunahme des BMI und Taillenumfangs bei ICT wurde in Quartile eingeteilt.

Ergebnisse: Patienten mit exzessiver Gewichtszunahme (im Mittel ca. 7 BMI-Einheiten) hatten nach einem Jahr und sechs Jahren um 0,6 bzw. 1,1\% höhere $\mathrm{HbA}_{1 \mathrm{c}}$-Werte als jene Patienten mit geringer Zunahme (im Mittel weniger als 3 BMI-Einheiten), trotz der etwas höheren Insulindosis. Mit der konventionellen Therapie nahm im gleichen Zeitraum der $\mathrm{HbA}_{1 \mathrm{c}}$ jener Patienten mit exzessiver Gewichtszunahme um 0,5\% ab. Komponenten des metabolischen Syndroms wie Taillenumfang, Blutdruck und

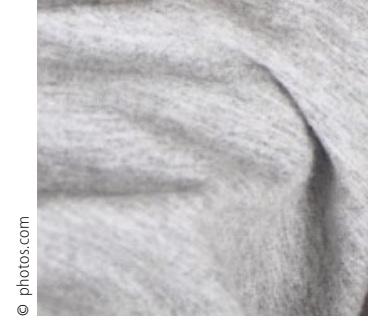

Die Anzeige der Waage sollte bei intensivierter Insulintherapie möglichst nicht in Richtung Adipositas klettern, um $\mathrm{Hb} A_{10}$, Triglyzeride und Blutdruck im Lot halten zu können.

Triglyzeride verschlechterten sich mit einer ICT bei Patienten mit exzessiver Gewichtszunahme. Das HDL-Cholesterin stieg aus nicht erklärbaren Gründen an. Je deutlicher das Gewicht in einem Jahr bzw. sechs Jahren anstieg, desto größer war die IMT an den Karotiden. Die IMT korrelierte mit traditionellen kardiovaskulären Risikofaktoren, nicht aber mit nicht traditionellen wie Apoprotein B, LDL-Partikelgröße, Lp(a) und Nicht-HDL-Cholesterin. Am engsten war die IMT mit einer exzessiven Gewichtszunahme korreliert. Ähnlich wie die IMT war der Kalziumscore mit ICT bei jenen am höchsten, die am stärksten zunahmen.

Schlussfolgerungen: Diese große Studie legt überzeugend dar, dass sich bei exzessiver Gewichtszunahme mit einer ICT eine abdominale Adipositas und eine Insulinresistenz einstellt; Blutdruck und Lipide sowie Komponenten des metabolischen Syndroms verschlechtern sich. Die größte IMT und der höchste Kalziumscore wurden bei Personen mit starker Gewichtszunahme festgestellt.
Purnell JQ, Zinman B, Brunzell JD et al. The effect of excess weight gain with intensive diabetes mellitus treatment on cardiovascular disease risk factors and atherosclerosis in type 1 diabetes mellitus. Circulation 2013;127:180-7.

\section{-Kommentar von Prof. Dr. med. Alfred Wirth}

\section{Aber vielleicht nicht bei allen Insulinarten?}

Eine intensive Insulintherapie reduziert bei Typ-1-Diabetikern nicht nur mikrovaskuläre Komplikationen, sondern auch - im Unterschied zu Typ-2-Diabetikern - makrovaskuläre Folgekrankheiten. Die vorliegende Studie zeigt erstmals, dass eine subklinische Arteriosklerose am besten verhindert werden kann, wenn die Gewichtszunahme so gering wie möglich ist. Faktoren, die dafür bedeutsam sind, können dem metabolischen Syndrom zugeordnet werden.

Was ist aus der Studie zu lernen? Die gesundheitlich beste intensive Insulintherapie bei Typ-1-Diabetikern ist diejenige, die mit der geringsten Gewichtssteigerung einhergeht. Erreichbar ist das mit einer niedrigen Insulindosis.

$\mathrm{Ob}$ auch die Insulinart von Bedeutung ist, ist Gegenstand der Forschung. Was zur klinischen Beurteilung fehlt, sind Ergebnisse zur Gewichtszunahme hinsichtlich klinischer Endpunkte und der Mortalität. Das will die EDIC-Studie jedoch schon bald liefern.

Prof. Dr. med. Alfred Wirth

Bad Rothenfelde 